\title{
New insights into the acetaminophen hepatotoxicity research
}

\begin{abstract}
Acetaminophen (APAP) toxicity is the leading cause of drug-induced acute liver failure (ALF) in the developed countries; however the underlying mechanisms of APAP toxicity are still not clear. Massive hepatocyte necrosis is the predominant feature of APAP hepatotoxicity; the liver regeneration is the vital process for survival after the toxic insult and many factors can influence liver repair. Emerging evidence shows that inflammation improves liver regeneration during the late phase of APAP hepatotoxicity; enhanced NF-kB activation, an important regulator of inflammation, is associated with improved liver recovery in APAP overdose; some widely used methods are not reliable to reveal liver regeneration in APAP toxicity research. These results indicate that the underlying mechanism of APAP hepatotoxicity is different from what currently well-accepted ones in which, inflammation is frequently considered to be responsible for liver tissue damage, and it is necessary to re-assess the underlying mechanism of liver regeneration in APAP overdose. This manuscript focuses on some new insights into the APAP toxicity research.
\end{abstract}

Keywords: Acetaminophen, Hepatotoxicity, Inflammation, Regeneration, NF-kB
Volume I Issue I - 2014

\author{
Runkuan Yang, ${ }^{1,2,3}$ Tor Inge Tonnessen, ${ }^{3,4}$ Sujun \\ $\mathrm{Li}^{5}{ }^{5}$ Jyrki Tenhunen ${ }^{1,6}$ \\ 'Department of Intensive Care Medicine, Tampere University \\ Hospital, University of Tampere, Finland \\ ${ }^{2}$ Department of Critical Care Medicine, University of Pittsburgh \\ Medical School, USA \\ ${ }^{3}$ Department of Emergencies and Critical Care, Oslo University \\ Hospital, Norway \\ ${ }^{4}$ Institute for Clinical Medicine, University of Oslo, Norway \\ ${ }^{5}$ Department of Health Care, Beijing Science and Technology \\ University Hospital, China \\ ${ }^{6}$ Department of Surgical Science, Anesthesiology and Intensive \\ Care Medicine, Uppsala University, Sweden
}

Correspondence: Runkuan Yang, Department of Intensive Care Medicine, University of Tampere, Medical School, 10 Bio katu,Tampere 330I4, Finland, Email bobyangr@yahoo.com

Received: May 15, 2014 | Published: May 30, 2014
Abbreviations: APAP; Acetaminophen, ALF; Acute Liver Failure, NAPQI; N-Acetyl-p-Benzoquinone imine, GSH; Glutathione, KCs; Kupffer Cells, IMs; Infiltrating Macrophages, LSEC; Liver Sinusoidal Endothelial Cell, RLS; Ringer's Lactate Solution, EP; Ethyl Pyruvate, BT; Bacterial Translocation, BrdU; 5-Bromo-2Deoxyuridine, PCNA; Proliferating Cell Nuclear Antigen, NAC; N-Acetyl-Cysteine, ROS; Reactive Oxygen Species, SIRS; Systemic Inflammatory Response Syndrome, MOF; Multiple Organ Failure

\section{Introduction}

Acetaminophen overdose is the major cause of acute liver failure (ALF) in the developed countries. ${ }^{1}$ The APAP hepatotoxicity is triggered by a highly reactive metabolite, $\mathrm{N}$-acetyl-p-benzoquinone imine (NAPQI), which depletes glutathione (GSH) and initiates mitochondrial oxidative stress ${ }^{2,3}$ this leads to the collapse of the mitochondrial membrane potential, the latter diminishes the mitochondrial capacity to synthesize ATP, ${ }^{4}$ and ATP depletion leads to massive hepatocyte necrosis, which is the predominant feature of APAP hepatotoxicity, ${ }^{4,5}$ and liver repair is the key to survival after the toxic insult. ${ }^{6-8}$ Currently the underlying mechanisms of the APAP toxicity are still not completely understood. It is well accepted that inflammation contributes to early liver injury in APAP overdose; however, emerging evidence is showing that inflammation also improves the liver regeneration during the late phase of APAP hepatotoxicity, ${ }^{9-13}$ and NF-kB, a master regulator of inflammation, plays an important role in modulating hepatic regeneration in APAP overdose. ${ }^{10,13,14}$ Since hepatocytes are mostly in a quiescent state $(\mathrm{G} 0),{ }^{8}$ pro-inflammatory cytokines such as TNF- $\alpha$ and IL- $6^{8,15,16}$ are needed to prime hepatocytes, this process makes cells more responsive to growth factors. The exposure to hepatocyte growth factor results in the expression of cell cycle proteins and the induction of cyclin D1 is the most reliable marker for cell cycle (G1 phase) progression in hepatocytes. Once hepatocytes express cyclin D1, they have passed the G1 restriction point and are committed to DNA replication. ${ }^{8}$
Many factors including nutrients and metabolic status can influence liver regeneration. ${ }^{8,15,16}$ After the loss of a large number of parenchymal cells, the metabolic work of surviving hepatocytes is increased and more ATP is needed for maintaining homeostasis and regeneration in APAP overdose. ${ }^{15}$

This manuscript focuses on some new insights into the APAP hepatotoxicity research.

The roles of Kupffer cells and liver sinusoidal endothelial cells in APAP hepatotoxicity.

Kupffer cells (KCs) are the most abundant mononuclear phagocytes in the body and a predominant source of inflammatory cytokines released into the systemic circulation. ${ }^{17}$ Kupffer cells play an important role in APAP overdose, ${ }^{12,18}$ the depletion of KCs confers protection at early time point ${ }^{18}$ but can lead to more severe injury at later time point during APAP hepatotoxicity. ${ }^{12}$ These results indicate that it is possible that KCs might play a "dual" role in APAP overdose: the classically activated M1 and alternatively activated M2 populations are proliferating and/ or migrating into the liver, ${ }^{11,19,20}$ and the M1 subset macrophages might play hepatotoxic role at early stage of APAP toxicity, while the M2 subset macrophages likely play hepatoprotective role at late phase of APAP-induced liver injury. ${ }^{19}$ This notion is further supported by the other APAP toxicity studies $^{9,21}$ in which the anti-inflammatory agent ethyl pyruvate (EP) reduces liver injury at early phase but impairs hepatic regeneration at late phase ${ }^{9}$ while Ringer's lactate, the pro-inflammatory solution, improves liver recovery at late phase. ${ }^{10}$ Emerging evidence shows that the combined absence of hepatic resident macrophages (KCs) and infiltrating macrophages (IMs) results in a marked delay in liver repair, and this delay is not due to impaired hepatocyte proliferation but rather prolonged vascular leakage, which is caused by APAPinduced liver sinusoidal endothelial cell (LSEC) injury. KCs express an array of angiogenic factors and induce LSEC proliferation and migration, ${ }^{21}$ suggesting that KCs play an important role in liver blood vessel repair during APAP hepatotoxicity. 


\section{The role of neutrophils in APAP liver injury}

The role of neutrophils in APAP toxicity is currently controversial. Neutrophils have been shown to contribute to liver injury in APAP overdose; ${ }^{22}$ however, conflicting data are also reported that a significant reduction in neutrophils accumulation shows no protection against APAP toxicity. ${ }^{23} \mathrm{New}$ evidence shows that pro-inflammatory therapy, the Ringer's lactate solution (RLS), does not worsen liver injury at early phase but improves liver repair at the late phase of APAP overdose, and the beneficial effect is not associated with neutrophil activity (MPO) level ${ }^{10}$ the anti-inflammatory agent ethyl pyruvate (EP) reduces early stage liver injury but impairs liver regeneration at the late phase of APAP toxicity; the early protective effect is associated with decreased hepatic MPO level; however, the late detrimental effect is not associated with hepatic MPO concentrations. These results indicate that neutrophils might contribute to early liver injury but do not play a major role in liver regeneration during APAP toxicity.

\section{The role of TNF-a in APAP toxicity}

Currently the role of TNF-a in APAP overdose is controversial. The pro-inflammatory mediator TNF-a has been demonstrated to promote tissue damage during APAP toxicity: ${ }^{24-26}$ however, TNF- $\alpha$ is also reported as an important pro-regenerative cytokine, which can prime hepatocytes to facilitate liver regeneration. ${ }^{8,27,28}$ New evidence shows that pro-inflammatory Ringer's lactate solution (RLS) increases early hepatic tissue TNF-a concentration but does not worsen the early liver injury in APAP overdose; RLS increases serum TNF-a level at late phase and the increased serum TNF-a level is associated with improved liver recovery in APAP overdose. ${ }^{10}$ The anti-inflammatory agent ethyl pyruvate (EP) reduces liver injury at early phase but impairs hepatocyte regeneration during the late phase of APAP hepatotoxicity, and the impaired late phase liver repair is associated with decreased serum TNF-a. ${ }^{9}$ Blockade of HMGB1 does not reduce early liver injury even though it decreases early hepatic TNF-a level during APAP toxicity; however, this therapy improves late phase liver regeneration, and this beneficial effect is associated with increased TNF-a level during the late phase of APAP hepatotoxicity. ${ }^{11}$ These new evidences suggest that TNF-a might contribute to early liver injury, but it is not a strong early injurious factor in APAP overdoses; early increased TNF-a might prime hepatocyte to facilitate the late phase liver regeneration; the increased TNF-a level at late time point is associated with improved liver repair during APAP hepatotoxicity.

\section{The role of HMGB I in APAP overdose}

APAP overdose induces massive hepatocyte necrosis, ${ }^{4,5,9,10}$ and necrotic tissue passively releases HMGB $1,{ }^{29-31}$ which is a ubiquitous nuclear protein secreted by immunocompetent cells, including monocytes, macrophages and neutrophils, and this highly conservative nuclear protein is an important late inflammatory mediator in sepsis. ${ }^{32}$ HMGB1 plays an important role in modulating inflammatory cascade in activated macrophages: HMGB1 stimulates macrophages to release TNF- $\alpha$ and IL-6, while HMGB1neutralizing antibody can block TNF- $\alpha$ release ${ }^{33,34}$ and knocking-out HMGB1 receptor can reverse IL-6 release $\left.{ }^{34}\right]$. Exogenous HMGB1 injection induces liver injury in normal mice; ${ }^{35}$ HMGB1 impairs hepatocyte regeneration and blockade of HMGB1 improves liver regeneration in mice subjected to APAP overdose; ${ }^{13}$ anti-HMGB1 treatment protects against APAP hepatotoxicity in rats ${ }^{36}$ in addition, HMGB 1 mediates gut bacterial translocation (BT) during APAP hepatotoxicity. ${ }^{11}$

\section{The role of NF-kB in APAP overdose}

$\mathrm{NF}-\mathrm{kB}$ is a master regulator of inflammation and the activation of $\mathrm{NF}-\mathrm{\kappa B}$ is linked strongly not only to the inflammatory response, ${ }^{32,37,38}$ but also to liver regeneration, ${ }^{8} \mathrm{NF}-\kappa \mathrm{B}$ is currently thought to play a major role in the initiation of liver regeneration after cell or tissue loss (such as partial hepatectomy). ${ }^{8,16} \mathrm{NF}-\kappa \mathrm{B}$ activation also induces increased expression of survival genes, including BCLXL and A1 in liver injury. ${ }^{39}$ Inhibition of NF- $\mathrm{\kappa B}$ after partial hepatectomy results in massive hepatocyte apoptosis worsens liver injury and decreases survival. ${ }^{40}$ There is evidence suggesting that the impact of APAP toxicity ensues, at least in part, by dramatic modulation of inflammatory and/ or regeneration programs, ${ }^{41}$ therefore, it is possible that in APAP overdose, enhanced NF- $\mathrm{kB}$ activation diverts intracellular pathways from those associated with inflammation and cell death to mechanisms linked to recruitment and activation of proregenerative programs, this notion is supported by following studies: enhanced NF-kB DNA binding is associated with improved liver recovery during the late phase of APAP hepatotoxicity; ${ }^{10,13}$ in contrast, decreased NF-kB DNA binding is associated with impaired liver regeneration. ${ }^{14}$ The role of NF-kB during the early injurious phase of APAP toxicity is still not clear.

The roles of 5-bromo-2-deoxyuridine (BrdU), proliferating cell nuclear antigen (PCNA) and HE stainings in APAP toxicity research

Currently the BrdU and PCNA immunohistochemistry stainings are well-accepted to reveal hepatic regeneration in liver injuries; however, these two methods are not reliable to detect liver regeneration during APAP hepatotoxicity in the commonly used C57/BL6 mice, because the specificity of the first antibody significantly influences the results: $:^{10,11,13,42,43}$ the PCNA (Invitrogen) kits mainly stain the nonparenchymal cells while the BrdU kits mainly stain hepatocyte; ${ }^{10,11,13}$ the animal species also markedly influence the results: $24 \mathrm{hrs}$ after in B6C3F1 mice ${ }^{42}$ and B6J129 SVF2 mice, ${ }^{43}$ however, BrdU and PCNA (Invitrogen) methods only show negative or occasional positive staining in C57/BL6 mice; ${ }^{10,11,44}$ more importantly, 48 hrs after APAP subjected to C57/BL6 mice, both PCNA (Invitrogen) and BrdU methods show that the extent of hepatic PCNA /BrdU expression depends mainly on the extent of liver damage, because the expression of hepatic PCNA / BrdU is significantly correlated with the area of hepatocyte necrosis for each mouse: larger size of necrosis has larger number of PCNA/BrdU positive cells, while improved liver repair with smaller necrotic size has smaller number of PCNA/BrdU positive cells, this is not conventionally expected..$^{10,11,44}$ In addition, the number of PCNA-positive non-parenchymal cells is 10 times larger than the number of BrdU-positive hepatocyte in C57/BL6 mice during APAP hepatotoxicity, ${ }_{11}^{11}$ suggesting that currently the non-parenchymal cells are likely underestimated, and the number of the non- parenchymal cell loss could be larger than the hepatocyte loss during APAP toxicity, the latter could explain the impaired immune function and the higher incidence of gram-negative infection during hepatotoxicity, ${ }^{11,45}$ further investigation is needed to focus on non parenchymal cell loss in APAP overdose.

Currently HE staining is not widely used to detect early hepatocyte regeneration; however, HE staining is reliable to reveal liver regeneration in C57/BL6 mice during APAP hepatotoxicity, ${ }^{11,13}$ and the pattern of hepatocyte regeneration revealed by HE staining does not match the liver proliferation revealed by BrdU/ PCNA methods, ${ }^{10,11,13}$ these results indicate that the conventional PCNA and BrdU methods are not reliable to reveal liver regeneration in C57/BL6 mice subjected to APAP overdose and other parameters are needed to detect liver repair in APAP toxicity. 


\section{The role of Cyclin DI in APAP overdose}

Regeneration ensures the replacement of necrotic cells and the full recovery of organ function. The induction of cyclin D1 is the most reliable marker for cell cycle (G1 phase) progression in hepatocytes, because once hepatocytes express cyclin D1, they have passed the G1 restriction point and are committed to DNA replication. ${ }^{8}$ Ringer's lactate solution (RLS) therapy and the anti-HMGB1 treatment enhance liver recovery at the late phase of APAP toxicity by increasing cyclin D1 expression; ${ }^{10,13}$ in contrast, the Ethyl pyruvate (EP) therapy and the prolonged treatment with $\mathrm{N}$-acetyl-cysteine (NAC) impair liver regeneration by decreasing cyclin D1 expression. ${ }^{9,14}$ These reports support the notion that cyclin D1 is reliable to reveal liver regeneration during APAP hepatotoxicity.

\section{The role of NAC therapy in APAP overdose}

Currently $\mathrm{N}$-acetyl-cysteine is the antidote for APAP toxicity, ${ }^{46}$ and it is commonly used for more than 24 hours in many medical centres. However, this antidotal therapy is effective only for patients who present within hours of an acute overdose, and is less effective for delayed cases. ${ }^{46,47}$ In addition, prolonged (longer than $24 \mathrm{hrs}$ ) treatment with NAC could be toxic and delays liver regeneration in APAP hepatotoxicity, and the detrimental effect is associated with reduced NF-kB DNA binding and decreased cyclin D1 expression. ${ }^{14}$

\section{The anti-and pro-inflammatory therapies in APAP toxicity}

EP is a potent anti-inflammatory agent and a reactive oxygen species (ROS) scavenger. ${ }^{48,49}$ EP inhibits LPS-stimulated macrophages to release TNF-a, IL-6 and HMGB1;32 EP also protects against liver injury in the following models: acute alcoholic hepatitis, ${ }^{50}$ hemorrhagic shock, ${ }^{51}$ sepsis, ${ }^{52}$ acute extrahepatic obstruction, ${ }^{53}$ and acute necrotizing pancreatitis. ${ }^{54}$ EP reduces liver injury at 24 hours but impairs liver regeneration at $48 \mathrm{hrs}$ during APAP hepatotoxicity, and the late detrimental effect is associated with decreased serum TNF-a concentration and reduced cyclin D1 expression in liver tissue.

Ringer's lactate solution (RLS) is a frequently used resuscitative fluid, which has been shown to increase serum IL-6, IL- $8^{55,56}$ and TNF- $\alpha^{5,57}$ in patients and experimental animals; in addition, RLS can provide lactate as an alternative metabolic fuel. ${ }^{58-64}$ "Lactic acid" was thought to be responsible for tissue damage, and as a consequence, lactate is frequently considered to be a "toxic" compound. These concepts are now being re-examined as metabolic evidence has emerged in favour of lactate reassessment. ${ }^{65,66}$ Lactate provides a satisfactory alternative to glucose as the primary energy in brain tissue during recovery from hypoxia, ${ }^{67,68}$ and lactate infusion can improve the recovery of neuron damage following brain injury. ${ }^{63}$ Moreover, lactate improves cardiac efficiency during shock, and it has recently been shown that lactate deprivation during shock impairs heart metabolism. ${ }^{69}$ These evidences indicate that lactate can be used as an energy substrate and resuscitative fluid to improve liver repair in APAP overdose, and this hypothesis has been confirmed. ${ }^{10}$ The pro-inflammatory RLS solution improves liver regeneration in APAP toxicity, the beneficial effect is associated with an augmented NF- $\mathrm{BB}$ DNA binding, increased hepatic cyclin D1 expression, and the increased pro-regenerative cytokine TNF- $\alpha$ concentrations, which might prime hepatocyte to facilitate liver regeneration.

\section{The role of gut barrier dysfunction in APAP toxicity}

During APAP hepatotoxicity, the gut mucosa is significantly injured, the intestinal mucosal permeability is markedly increased, and the loss of microvillus epithelial cell line is confirmed by pathology. APAP overdose induces evident gut bacterial translocation (BT) ${ }^{11}$ which might be responsible for the high incidence of intestinal gram-negative bacterial infection during hepatotoxicity, and BT might trigger the systemic inflammatory response syndrome (SIRS), which can lead to multiple organ failure (MOF) and mortality in acute liver failure (ALF). ${ }^{11,45}$ Blockade of HMGB1 reduces $85 \%$ of BT but does not decrease gut mucosal permeability during APAP hepatotoxicity, ${ }^{11}$ this indicates that BT is mediated (at least partly) by HMGB1, and neutralization of HMGB1 might present a novel therapy to prevent MOF from BT during APAP hepatotoxicity.

\section{Conclusion}

Inflammation contributes to early liver injury but improves liver repair during APAP hepatotoxicity, and anti-inflammation therapy at late phase is not beneficial. TNF- $\alpha$ facilitates liver regeneration in APAP overdose. NF-kB modulates liver regeneration at the late phase of APAP toxicity. Traditional PCNA and BrdU staining methods are not reliable to reveal the extent of liver regeneration in commonly used C57/BL6 mice during APAP toxicity; in contrast, cyclin D1 is a reliable parameter for liver regeneration in APAP overdose. Prolonged treatment with NAC is potentially toxic and delays liver recovery in APAP overdose. HMGB1 impairs hepatocyte regeneration and mediates BT during APAP hepatotoxicity.

\section{Acknowledgements}

This investigation was partly supported by Sigrid Juselius Funding in Finland.

\section{Conflicts of Inetrset}

None.

\section{References}

1. Lee WM. Acetaminophen and the U.S. Acute liver failure study group: lowering the risks of hepatic failure. Hepatology. 2004;40(1):6-9.

2. Nelson SD. Molecular mechanisms of the hepatotoxicity caused by acetaminophen. Semin Liver Dis. 1990;10(4):267-278.

3. CohenSD, KhairallahEA. Selective protein arylation and acetaminopheninduced hepatotoxicity. Drug Metab Rev. 1997;29(1-2):59-77.

4. Cressman DE, Greenbaum LE, DeAngelis RA, et al. Liver failure and defective Hepatocyte regeneration in interleukin-6 deficient mice. Science. 1996;274(5291):1379-1383.

5. Jaeschke $H$, Bajt ML. Intracellular signaling mechanisms of acetaminophen-induced liver cell death. Toxicol Sci. 2006;89(1):31-41.

6. Chanda S, Mehendale HM. Hepatic cell division and tissue repair: a key to survival after liver injury. Mol Med Today. 1996;2(2):82-89.

7. Mehendale HM. Tissue repair: an important determinant of final outcome of toxicant-induced injury. Toxicol Pathol. 2005;33(1):41-51.

8. Fausto N. Liver regeneration. J Hepatol. 2000;32(1 Suppl):19-31.

9. Yang R, Zou X, Koskinen ML, et al. Ethyl pyruvate reduces liver injury at early phase but impairs regeneration at late phase in acetaminophen overdose. Critical Care. 2012;16(1):R9.

10. Yang $\mathrm{R}$, Zhang $\mathrm{S}$, kajander $\mathrm{H}$, et al. Ringer's lactate improves liver recovery in a murine model of acetaminophen toxicity. $B M C$ Gastroenterol. 2011;11:125.

11. Yang R, Zou X, Tenhunen J, et al. Neutralization of HMGB1 is associated with bacterial translocation during acetaminophen hepatotoxicity. BMC Gastroenterol. 2014;14: 66. 
12. Ju C, Reilly TP, Bourdi M, et al. Protective role of Kupffer cells in acetaminophen-induced hepatic injury in mice. Chem Res Toxicol. 2002;15(12):1504-1513.

13. Yang R, Zhang S, Cotoia A, et al. High mobility group B1 impairs hepatocyte regeneration in acetaminophen hepatotoxicity. BMC Gastroenterol. 2012;12:45.

14. Yang R, Miki K, He X, et al. Prolonged treatment with $\mathrm{N}$-acetylcysteine delays liver recovery from acetaminophen hepatotoxicity. Crit Care. 2009;13(2):R55.

15. Akerman P, Coto P, Yang SQ, et al. Antibodies to tumor necrosis factoralpha inhibit liver regeneration after partial hepatectomy. Am J Physiol. 1992;263(4 Pt 1):G579-G585.

16. Cataldegirmen G, Zeng S, Feirt N, et al. Rage limits regeneration after massive liver injury by coordinated suppression of $\mathrm{TNF}-\alpha$ and $\mathrm{NF}-\kappa \mathrm{B}$ JEM. 2005;201(3):473-484.

17. Akbarshahi H, Rosendahl AH, Westergren-Thorsson G, et al. Acute lung injury in acute pancreatitis-awaiting the big leap. Respiratory Med. 2012;106(9):1199-1210.

18. Goldin RD, Ratnayaka ID, Breach CS, et al. Role of microphages in acetaminophen (paracetamol)-induced hepatotoxicity. J pathol. 1996;179(4):432-435.

19. Yang Q, Shi Y, He J, et al. The evolving story of macrophages in acute liver injury. Immunology Letters. 2012;147(1-2):1-9.

20. Laskin DL. Macrophages and inflammatory mediators in chemical toxicity: a battle of forces. Chem Res Toxicol. 2009;22(8):1376-1385.

21. You Q, Holt M, Yin H, et al. Role of hepatic resident and infiltrating macrophages in liver repair after acute injury. Biochemical Pharmacology. 2013;86(6):836-843.

22. Liu ZX, Han D, Gunawan B, et al. Neutrophils depletion protects agains murine acetaminophen hetatotoxicity. Hepatology. 2006;43(6):1220 1230 .

23. Bauer I, Vollmar B, Jaeschke H, et al. Transcriptional activation hemeoxygenase-1 and its functional significance in acetaminopheninduced hepatitis and hepatocellular injury in the rat. $J$ Hepatol. 2000;33(3):395-406.

24. Boess F, Bopst M, Althaus R, et al. Acetaminophen hepatotoxicity in tumor necrosis factor/lymphotoxin-alpha gene knockout mice. Hepatology. 1998;27(4):1021-1029.

25. Ishida Y, Kondo T, Tsuneyama K, et al. The pathogenic roles of tumor necrosis factor receptor $\mathrm{p} 55$ in acetaminophen-induced liver injury in mice. J Leukoc Biol. 2004;75(1):59-67.

26. Blazka ME, Wilmer JL, Holladay SD, et al. Role of inflammatory cytokines in acetaminophen hepatotoxicity. Toxicol Appl Pharmacol. 1995; 133(1):43-52.

27. Chiu H, Gardner CR, Dambach DM, et al. Role of tumor necrosis factor receptor $1(\mathrm{p} 55)$ in hepatocyte proliferation during acetaminopheninduced toxicity in mice. Toxicol Appl Pharmacol. 2003;193(2):218-227.

28. Yamada Y, Kirillova I, Peschon JJ, et al. Initiation of liver growth by tumor necrosis factor: deficient liver regeneration in mice lacking type 1 tumor necrosis factor receptor. Proc Natl Acad Sci. 1997;94(4):1441-1446.

29. Wang H, Li W, Goldstein R, et al. HMGB1 as a potential therapeutic target. Novartis Found Symp. 2007;280:73-85.

30. Yang H, Wang H, Czura CJ, et al. The cytokine activity of HMGB1. J Leukoc Biol. 2005;78(1):1-8.

31. Scaffidi P, MisteliT, Bianchi ME. Release of chromatin protein HMGB1 by necrotic cells triggers inflammation. Nature. 2002;418(6894):191-195.

32. Ulloa L, Ochani M, Yang H, et al. Ethyl pyruvate prevents lethality in mice with established lethal sepsis and systemic inflammation. Proc Natl Acad Sci. 2002;99(19):12351-12356.

33. Gazzar ME. HMGB1 modulates inflammatory responses in LPSactivated macrophages. Inflamm Res. 2007;56(4):162-167.

34. Yang H, Hreggvidsdottir HS, Palmblad K, et al. A critical cysteine is required for HMGB1 binding to toll-like receptor 4 and activation of macrophage cytokine release. PNAS. 2010;107(26):11942-11947.

35. Sappington PL, Yang R, Yang H, et al. HMGB1 B box increases the permeability of Caco-2 enterocytic monolayers and impairs intestina barrier function in mice. Gastroenterology. 2002;123(3):790-802.

36. Antoine DJ, Williams DP, Kipar A, et al. Diet restriction inhibits apoptosis and HMGB1 oxidation and promotes inflammatory cell recruitment during acetaminophen hepatotoxicity. $\mathrm{Mol} \mathrm{Med}$. 2010;16(11-12):479-490.

37. Tsung A, Sahai R, Tanaka H, et al. The nuclear factor HMGB1 mediates hepatic injury after murine liver ischemia-reperfusion. JEM. 2005;201(7):1135-1143.

38. Luedde T, Schwabe RF. NF-kB in the liver-linking injury, fibrosis and hepatocellular carcinoma. Nat Rev Gastroenterol Hepatol. 20111;8(2):108-118

39. Malhi H, Gores GJ, Lemasters JJ. Apoptosis and necrosis in the liver: a tale of two deaths? Hepatology. 2006;43(2 Suppl 1): S31-S44.

40. Iimuro Y, Nishiura T, Hellerbrand C, Behrns KE, Schoonhoven R, et al. (1998) NF kappa B prevents apoptosis and liver dysfunction during liver regeneration. J Clin Invest 101(4): 802-811.

41. Kaplowitz N. Acetaminophen hepatotoxicity. What do we know, what don't we know, and what do we do next? Hepatology. 2004;40(1):23-26.

42. Milesi-Halle A, McCullough S, Hinson JA, et al. Echinomycin decreases induction of vascular endothelial growth factor and hepatocyte regeneration in acetaminophen toxicity in mice. Basic Clin Pharmacol Toxicol. 2012;110(4):327-334.

43. Gardner CR, Gray JP, Joseph LB, et al. Potential role of caveolin-1 in acetaminophen-induced hepatotoxicity. Toxicol Appl Pharmacol. 2010;245(1):36-46.

44. Vaquero J, Belanger M, James L, et al. Mild hypothermia attenuates liver injury and improves survival in mice with acetaminophen toxicity. Gastroenterology. 2007;132(1):372-383.

45. Kasravi FB, Wang LQ, Wang XD, et al. Bacterial translocation in acute liver injury induced by $\mathrm{D}$-galactosamine. Hepatology. 1996;23(1):97-103.

46. Whyte IM, Francis B, Dawson AH. Safety and efficacy of intravenous N- acetylcystine for acetaminophen overdose: analysis of the Hunter Area Toxicity Service (HATS) database. Curr Med Res Opin. 2007;23(10):2359-2368.

47. Kerr F, Dawson A, Whyte IH, et al. The austrilian clinical toxicology investigators collaboration Randomized trial of different loading infusion rates of N-acetylcystine. Annals Emerg Med. 2005;45(4):402-408.

48. Fink MP. Ethyl pyruvate: a novel treatment for sepsis. Curr Drug Targets. 2007;8(4):515-518.

49. Fink MP. Ethyl pyruvate: a novel anti-inflammatory agent. J Intern Med. 2007261(4):349-362.

50. Yang R, Han X, Delude RL, et al. Ethyl pyruvate ameliorates acute alcoholic-induced liver injury and inflammation in mice. J Lab Clin Med. 2003;142(5):322-331.

51. Yang R, Gallo DJ, Baust JJ, et al. Ethyl pyruvate modulates inflammatory gene expression in mice subjected to hemorrhagic shock. Am J Physiol Gastrointest Liver Physiol. 2002;283(1):G212-G221. 
52. Sappington PL, Han X, Yang R, et al. Ethyl pyruvate ameliorates intestinal epithelial barrier dysfunction in endotoxemic mice and imunostimulated Caco-2 enterocytic monolayers. J Pharmacol Exp Therap. 2003;304(1):464-476.

53. Yang R, Uchiyama T, Watkins SK, et al. Ethyl pyruvate reduces liver injury in a murine model of extrahepatic cholestasis. Shock. 2004;22(4): $369-375$.

54. Yang R, Uchiyama T, Alber SM, et al. Ringer's ethyl pyruvate solution ameliorates distant organ injury in a murine model of acute necrotizing pancreatitis. Crit Care Med. 2004;32(7):1453-1459.

55. Lang K, Suttner S, Boldt J, et al. Volume replacement with HES 130/0.4 may reduce the inflammatory response in patients undergoing major abdominal surgery. Can J Anaesth. 2003;50(10):1009-1016.

56. Tamayo E, Alvarez FJ, Alonso O, et al. The inflammatory response to colloids and crystalloids used for pump priming during cardiopulmonary bypass. Acta Anaesthesiol Scand. 2008;52(9):1204-1212.

57. Deree J, Loomis WH, Wolf $\mathrm{P}$, et al. Hepatic transcription factor activation and proinflammatory mediator production is attenuated by hypertonic saline and pentoxifylline resuscitation after hemorrhagic shock. J Trauma. 2008;64(5):1230-1238.

58. King $\mathrm{P}$, Kong MF, Parkin $\mathrm{H}$, et al. Intravenous lactate prevents cerebral dysfunction during hypoglycaemia in insulin-dependent diabetes mellitus. Clin Sci (Lond). 1998;94(2):157-163.

59. Maran A, Crepaldi C, Trupiani S, et al. Brain function rescue effect of lactate following hypoglycaemia is not an adaptation process in both normal and type I diabetic subjects. Diabetologia. 2000;43(6):733-741.

60. Maran A, Cranston I, Lomas J, et al. Protection by lactate of cerebral function during hypoglycaemia. Lancet. 1994;343(8888):16-20.
61. Schurr A. Lactate: the ultimate cerebral oxidative energy substrate? $J$ Cereb Blood Flow Metab. 2006;26(1):142-152.

62. Schurr A, Payne RS, Miller JJ, et al. Brain lactate is an obligatory aerobic energy substrate for functional recovery after hypoxia: further in vitro validation. J Neurochem. 1997;69(1):423-426.

63. Rice AC, Zsoldos R, Chen T, et al. Lactate administration attenuates cognitive deficits following traumatic brain injury. Brain Res. 2002;928(1-2):156-159.

64. Philp A, Macdonald AL, Watt PW. Lactate-a signal coordinating cell andsystemic function. J Exp Biol. 2005;208(Pt 24):4561-4575.

65. Leverve XM. Energy metabolism in critically ill patients: lactate is a major oxidizable substrate. Curr Opin Clin Nutr Metab Care. 1999;2(2):165-169.

66. Leverve XM, Mustafa I, Peronnet F. Pivotal role of lactate in aerobic metabolism. Year book of intensive care and emergency medicine. J V Springer, Berlin, 1998; pp. 588-596.

67. Schurr A. Lactate, glucose and energy metabolism in the ischemic brain (Review). Int J Mol Med. 2002;10(2):131-136.

68. Schurr A, Payne RS, Miller JJ, et al. Brain lactate, not glucose, fuels the recovery of synaptic function from hypoxia upon reoxygenation: an in vitro study. Brain Res. 1997;744(1):105-111.

69. Levy B, Mansart A, Montemont C, et al. Myocardial lactate deprivation is associated with decreased cardiovascular performance, decreased myocardial energies, and early death in endotoxic shock. Intensive Care Med. 2007;33(3):495-502. 